\title{
Levels and speed of processing effects on word analysis
}

\author{
DANIEL B. KAYE \\ University of California, Los Angeles, California \\ and \\ SCOTT W. BROWN \\ University of Connecticut, Storrs, Connecticut
}

\begin{abstract}
Previous work on semantic priming effects has suggested that priming is contingent upon semantic analysis of the prime stimulus. In the present series of experiments, subjects in Grade 3 , Grade 6, and college performed variants of a priming task in which semantic (lexical decision) as well as fast (case judgment) and slow (letter search) nonsemantic levels of processing were required for both prime and target stimuli. Contrary to a levels of processing hypothesis, context effects were not simple functions of the level at which the prime was processed. Priming effects were found with a slow nonsemantic prime task (letter search), but generally not with a fast nonsemantic analysis of the prime (case judgment). The form of the priming effect when the prime was processed semantically by a lexical decision depended on the relationship between prime and target processing: a switch from semantic prime processing to nonsemantic target processing produced Stroop-like interference, with other combinations of prime and target processing producing facilitation. By using a series of discrimination, focusing, and classification tasks in a Garner (1978) paradigm, it was possible to determine how subjects were processing the semantic and nonsemantic dimensions, and these perceptual strategies were compared across educational levels to account for the priming effects. Our results suggest that context effects need to be understood in terms of the speeds of processing of different codes of information inherent in words.
\end{abstract}

The semantic priming ("context") effect in word recognition refers to the now well-established finding that the semantic relationship between prime and target words can affect the speed at which the target word is processed (Meyer, Schvaneveldt, \& Ruddy, 1975). Typically, the priming effect has been accounted for by semantic processing of the context. In particular, M. C. Smith (1979) has hypothesized that the level of processing of the prime is the critical factor in the generation of context effects. Our experiments support a modified levels of processing theory of semantic context effects. The theory acknowledges the role of two factors: (1) the interaction between levels of processing of the prime and

This research was supported in part by UCLA Academic Senate Grant No. 3736 and by Biomedical Research Support Grant No. 4-52342824739. Portions of this paper were presented at the meeting of the Society for Research in Child Development, Detroit, April 1983. We extend our appreciation to the staff and students of the Seeds University Elementary School, UCLA, and to the staff and students of Coventry Public Schools, Coventry, Connecticut, for their cooperation; to Edward Herskovits, Lisa Fonseca, Ginny Bonnefil, Chen Qi, and Lephuong Phi for subject testing and data analysis; to Lisa Kahan, Janice Lawry, and Wendell Jeffrey for helpful comments on earlier drafts; and to Roger Schvaneveldt, Curtis Becker, and Roberta Klatzky for insightful and constructive criticism. Send reprint requests to D. B. Kaye, Department of Psychology, University of California, 405 Hilgard Avenue, Los Angeles, CA 90024. target stimuli, and (2) the relative speeds of processing semantic and nonsemantic levels of the prime and target stimuli.

\section{Levels of Processing}

Recently, attention has been given to the type of context effects produced by different levels of prime processing. M. C. Smith (1979) tested the hypothesis that context effects are contingent upon lexical analysis (as one type of semantic analysis) of the prime stimulus by asking whether context effects would be found if subjects' attention was redirected from a lexical analysis of the prime to a letter-by-letter prelexical analysis. She found, with adult subjects, that contextual facilitation was possible when subjects conducted a semantic analysis of the prime (without a response) followed by a letter search of the target, but not when they performed a letter search of both the prime and the target. Smith concluded tentatively that semantic analysis of the prime is necessary for the context effect.

A more complete test of the levels of processing hypothesis was conducted in a subsequent study (M. C. Smith, Theodor, \& Franklin, 1983). In Experiment 1 of that study, subjects in each of five groups were required to execute a lexical decision of the target stimulus, with a different task required for the prime stimulus in each group. Three of these tasks were modeled after typical 
levels of processing experiments (e.g., Craik \& Tulving, 1975): (1) visual analysis-subjects were asked to detect whether a star preceded the prime, (2) phonemic analysis-subjects were asked to count the number of syllables in the prime, and (3) semantic analysis-subjects were asked to classify the prime as a living or not-living thing. Two other tasks were included: (4) prime letter search-similar to the prelexical condition in M. C. Smith (1979), and (5) silent reading of the prime stimulus, as in the typical lexical decision task.

Results of the M. C. Smith et al. (1983) study supported the levels of processing hypothesis of M. C. Smith (1979). The amount of facilitation was dependent on the depth of processing of the prime: neither the letter search nor visual analysis conditions resulted in any facilitation of the target lexical decision, but the phonemic, silent reading, and semantic decision conditions did result in facilitation, with the greatest facilitation in the latter condition. Thus, the deeper the level of processing of the prime, the more facilitation of the target lexical decision.

Additional support for the levels of processing explanation derives from experiments reported by Henik, Friedrich, and Kellogg (1983). In their first experiment, they used a target lexical decision task but varied the level of prime processing by asking subjects to conduct a letter search or to name the prime stimulus. Naming produced a significant $34-\mathrm{msec}$ related word advantage, but letter search yielded equivalent reaction times (RTs) for related and unrelated word pairs. In subsequent experiments, Henik et al. found that the priming effect was in the form of interference when color naming was the target task, as the response was incompatible with the semantic response required of the prime (i.e., Stroop-like interference).

But other research indicates that it may be too simple to argue that the nominal level of prime processing is sufficient to account for context effects. There is some evidence that context effects can be found even when the nominal level of prime processing is not semantic. The phonemic analysis condition produced a facilitation effect in M. C. Smith et al. (1983), and DonnenwerthNolan, Tanenhaus, and Seidenberg (1981) demonstrated a facilitation effect in a rhyme monitoring task. Marcel (1983) and Fowler, Wolford, Slade, and Tassinary (1981) have reported evidence for semantic priming effects in lexical decision tasks when prime stimuli are not perceived consciously (i.e., when they are presented at rates below detection level). These results can be accommodated by a modified levels of processing hypothesis: It is possible that semantic access may occur without perceptual awareness or intentional semantic analysis; in this event, priming may still be due to semantic processing of the prime, albeit at an unconscious level. One possibility to explore is that semantic context effects may result even with nominally nonsemantic levels of prime processing when the nonsemantic analysis is more time-consuming than a semantic analysis. For example, if the letter code takes longer to access than the lexical code, then access to a prime's meaning may occur before letter search of the prime is completed.

\section{Speed of Processing}

This speed of processing aspect of priming is highlighted by developmental investigations of priming effects. For example, Stanovich (1980) has described an interactive-compensatory model, in which younger or poorer readers compensate for their relatively slow word recognition processes by using semantic context to a greater extent to facilitate recognition of target words.

That the use of context diminishes with age and ability has been shown repeatedly (Schvaneveldt, Ackerman, \& Semlear, 1977; Schwantes, Boesl, \& Ritz, 1980; Stanovich, West, \& Feeman, 1981). Stanovich et al. demonstrated that the general decrease in the context effect begins as early as the second half of Grade 2, about the same time that word recognition becomes faster. A general prediction of the compensatory view is that context effects will be largest when visual analysis is made difficult, as when words are visually degraded (Meyer et al., 1975; Stanovich \& West, 1979) or when especially difficult or unfamiliar words are used as stimuli (Stanovich et al., 1981). Because the speed (and accuracy) of word recognition increases steadily for many years (Doehring, 1976), even beyond the initial development of automatic word recognition skill, context effects would be expected to diminish steadily, as they appear to do.

The series of three experiments reported in this paper was designed to further test the hypotheses that context effects (1) are contingent upon semantic processing of the context information, and (2) are dependent upon the speed at which semantic and nonsemantic analyses are conducted. In particular, we predict that semantic context effects can be found even when the nominal analysis of the prime is nonsemantic, if the nonsemantic analysis takes enough time so that a semantic analysis can also occur. Context effects would be expected, regardless of the level of target processing, if the prime analysis takes long enough for semantic access to occur, although the form of the context effect will depend on the compatibility of the target response with the prime response. If a semantic prime response is followed by a nonsemantic target response, then inhibition is predicted, as in the case of the Stroop-like effect found by Henik et al. (1983); otherwise, facilitation is expected.

We extended the tests of M. C. Smith (1979; M. C. Smith et al., 1983) and Henik et al. (1983) by including a more complete set of tasks involving both semantic and nonsemantic analyses of both context and target stimuli. In Experiment 1, we tested our theory by using letter search and lexical decision tasks to determine whether context effects would be found with the slow nonsemantic task. In Experiment 2, we used a set of discrimination, focusing, and classification tasks (Garner, 1978, paradigm) to validate case judgment as a fast nonsemantic task. Furthermore, these tasks were used to determine whether subjects could process both the semantic and non- 
semantic codes in the word stimuli: Most theories of context effects assume that separate codes of the word are accessible and analyzable. Experiment 2 provided a direct test of the perceptual analysis of these stimuli. In Experiment 3 , we tested for diminished context effects with the fast nonsemantic task (case judgment) and the lexical decision task.

Furthermore, we sampled subjects in Grade 3, Grade 6, and college, which enabled us to test context effects as a function of naturally occurring increases in word processing speed, to test the similarity of perceptual analysis strategies at different ages, and to examine the relationship of these analysis strategies to context effects.

\section{EXPERIMENT 1}

To review, according to the levels of processing hypothesis (e.g., M. C. Smith, 1979), context effects are a function of the level of processing of the prime. This view predicts that context effects will be found when the context is processed semantically, and M. C. Smith (1979) demonstrated that priming effects are possible even when attention to the target is directed to nonsemantic levels of processing. On the other hand, the theory predicts an absence of context effects when letter search or some other nonsemantic response is required of the prime. Therefore, we varied the levels of prime and target processing orthogonally: There were four conditions derived from combinations of prime and target nonsemantic (letter search, or LS) and semantic (lexical decision, or LD) levels of processing.

Our results are organized about the level of prime processing, since it is essential to the levels theory. However, our results demonstrate that neither the prime nor the target level of processing alone is sufficient to account for context effects; rather, the relationship between prime and target levels of processing must be considered. Furthermore, the speed of processing of the letter and word information requisite for letter search and lexical decision responses is critical, and we present the age effects to support this speed of processing hypothesis.

\section{Method}

Subjects. The subjects were 32 middle-class suburban third graders (mean age $=9.17$ years, $S D=0.43$ ), 32 sixth graders (mean age $=12.08$ years, $\mathrm{SD}=0.42$ ), and 32 college undergraduates. Subjects from each academic level were assigned randomly to one of the four experimental conditions described below $(n=24$ for each condition). Three subjects from Grade 3 were dropped due to incomplete data or extremely high error rates-one from the LD/LD and two from the LS/LD condition.

Materials. The word stimuli were selected from a master list compiled in a preliminary normative study in which independent samples of third and sixth graders provided word associations for 450 high-frequency nouns ranging in length from 3 to 6 letters Imean Standard Frequency Index $(\mathrm{SFI})=58.44, \mathrm{SD}=4.48$; Carroll, Davies, \& Richman, 1971]. From the word association protocols, 30 noun pairs were selected for which frequency of response was high in Grades 3 and 6 . Word length was roughly equivalent for prime and target words selected. Words in other task conditions, for which high associates were not necessary, also were selected from the master list and were matched to the high associate conditions for word length and summed letter-positional frequency (SPF; Mayzner \& Tresselt, 1963). Nonwords were pronounceable pseudowords constructed from the remaining set of words such that the number of letters and SPFs were matched with those of the words in other conditions. Across conditions, the mean SPF was 1.565 for words and 1,544 for pseudowords $(\mathrm{p}>.05$, Fisher's LSD t test).

Apparatus. Stimuli in the lexical decision phase were presented on a Hitachi 9-in. black-and-white monitor controlled by a Polymorphic 8813 microcomputer ( 8080 processor). Timing was software-controlled, with accuracy to less than $1 \mathrm{msec}$ (Post, 1979). Subjects responded via one of two predesignated keys on the detachable keyboard, allowing for accurate control of the viewing distance and providing a comfortable response environment.

Design. Two between-subjects and five within-subjects conditions were included. Between-subjects conditions varied the level of processing required of the prime and target orthogonally (prime $\times$ target; letter search vs. lexical decision for each), resulting in four between-subjects groups: (1) lexical decision of both prime and target (LD/LD), (2) lexical decision of prime followed by letter search of target ( $\mathrm{LD} / \mathrm{LS})$, (3) letter search of both prime and target (LS/LS), and (4) letter search of prime followed by lexical decision of target (LS/LD). For all groups of subjects, the stimuli were the same. Five types of stimulus pairs were presented. In the related-words condition, 30 pairs of semantically related nouns were presented successively in a trial, the first as the prime and the second as the target. In the unrelated-words condition, 30 pairs of semantically unrelated nouns were presented. The remaining three conditions served as controls, with 30 trials each of pseudoword. word, word-pseudoword, and pseudoword-pseudoword pairs. The relatedness effect was a comparison of RTs in the related- and unrelated-word conditions. For each of the five conditions, 30 pairs were presented. Presentation order of pairs was randomized across the total set of 150 trials (30 pairs $\times 5$ conditions) ${ }^{1}$

Procedure. Subjects in all grades were tested individually. Each trial consisted of three parts: (1) A centrally located ready signal (**READY**) appeared for $1 \mathrm{sec}$, followed by clearing of the screen; (2) after a 500-msec interval, a prime stimulus replaced the ready signal at the center of the screen until the subject responded, at which time the screen was cleared; and (3) after a 600-msec interstimulus interval (ISI), the target stimulus appeared until the subject's second response. In groups for which a letter search was required, the letter to be detected was located one line above the center of the letter string to be searched. (Target letters were balanced by position within stimulus.) Subjects responded manually on both tasks. One block of 16 practice trials (repeated if necessary) preceded 15 blocks of 11 experimental trials (the first trial of each block was a warmup trial and data were not recorded). Instructions stressed speed and accuracy, and both were measured.

\section{Results}

Mean RTs and error rates for the correct lexical decision responses to target stimuli for the related- and unrelated-word conditions are presented in Table $1 .^{2}$ These means include only trials for which the prime response was also correct.

Semantic prime processing. As predicted by the levels of processing hypothesis, context effects were found when the prime was processed semantically. Related words were recognized more rapidly than unrelated words when a lexical decision was required about both the prime and target words $(\mathrm{LD} / \mathrm{LD})$ : These results comprise the standard semantic priming effect, although the effect for the college subjects was small. Results of tests on the differences were: $t(6)=2.87, \mathrm{p}<.03$ for Grade 3 subjects; $t(7)$ 
Table 1

Mean Reaction Times (and Error Rates) to Target Words by Grade Level and Condition-Experiment 1

\begin{tabular}{|c|c|c|c|c|c|c|c|}
\hline \multicolumn{2}{|c|}{ Task } & \multirow{2}{*}{$\begin{array}{l}\text { Grade } \\
\text { Level }\end{array}$} & \multicolumn{5}{|c|}{ Condition } \\
\hline Prime & Target & & \multicolumn{2}{|c|}{ Related } & \multicolumn{2}{|c|}{ Unrelated } & \multirow{2}{*}{$\frac{\text { Context }}{+96 \ddagger}$} \\
\hline \multirow[t]{3}{*}{ LD } & LD & 3 & 990 & (02) & 1086 & $(02)$ & \\
\hline & & 6 & 750 & (02) & 822 & $(02)$ & $+72 \ddagger$ \\
\hline & & C & 499 & (01) & 511 & $(01)$ & $+12 \dagger$ \\
\hline \multirow[t]{3}{*}{ LD } & LS & 3 & 1416 & (02) & 1339 & (03) & $-77 \dagger$ \\
\hline & & 6 & 967 & (04) & 933 & (07) & $-34 \ddagger$ \\
\hline & & C & 787 & (03) & 748 & (03) & $-39 \S$ \\
\hline \multirow[t]{3}{*}{ LS } & LS & 3 & 1333 & (05) & 1406 & (05) & $+73 \dagger$ \\
\hline & & 6 & 957 & (02) & 1014 & (04) & $+57 \S$ \\
\hline & & C & 668 & (03) & 672 & (05) & +4 \\
\hline \multirow[t]{3}{*}{ LS } & LD & 3 & 1103 & (17) & 1155 & (24) & $+52 \dagger$ \\
\hline & & 6 & 918 & (04) & 940 & (08) & $+22 \dagger$ \\
\hline & & C & 532 & (08) & 556 & (11) & $+24 \ddagger$ \\
\hline
\end{tabular}

Note-RTs in msec; error rates in parentheses to nearest hundredths, decimals omitted. $L D=$ lexical decision; $L S=$ letter search; $C=$ college. ${ }^{*}$ Context effect $=$ unrelated $R T-$ related $R T . \dagger p<.15$. $\ddagger p<.05 . \$ p<.01$.

$=1.96, \mathrm{p}<.05$ for Grade 6 subjects; and $\mathrm{t}(7)=1.24$, $.10<\mathrm{p}<.15$ for college subjects (all one-tailed Fisher LSD tests).A related-word advantage was shown by 17 of 23 subjects (over grades) ( $p<.02$ by normal approximation to binomial probability; Hays, 1974, pp. 304 ff.), with no difference in distributions among the grades.

However, when the prime task was lexical decision and the target task was letter search (LD/LS), the priming effect took a different form: Related target words were responded to more slowly than unrelated target words at every grade level (see table 1) [for Grade 3 subjects, $t(7)$ $=1.52, .05<\mathrm{p}<.10$; for Grade 6 subjects, $\mathrm{t}(7)=$ $2.15, \mathrm{p}<.05 ;$ for college subjects, $\mathrm{t}(7)=3.81$, $p<.005]$. This unrelated-word advantage was shown by 19 of the 24 third graders, sixth graders, and college subjects $(p<.003$ by normal approximation to the binomial test).

Nonsemantic prime processing. It would appear that the key prediction of the levels of processing hypothesis is the absence of context effects with nonsemantic prime processing. But we found facilitation effects with prime letter search, independent of the level of processing of the target. When a letter search was conducted on both the prime and target (LS/LS), a context facilitation effect was found in Grade 3 and Grade 6 subjects, but not in college subjects [for Grade 3 subjects, $t(7)=1.70$, $.05<\mathrm{p}<.10$; for Grade 6 subjects, $\mathrm{t}(7)=4.07$, $\mathrm{p}<.005]$. The related-word advantage was demonstrated by 13 of 16 children ( $p<.01$, by the binomial test).

When the letter search was conducted on the prime and lexical decision on the target (LS/LD), all groups were faster with related-word pairs than with unrelated-word pairs [for Grade 3 subjects, $t(5)=1.77, .05<\mathrm{p}<.10$; for Grade 6 subjects, $t(7)=1.27, .10<p<.15$; for college subjects, $t(7)=2.23, \mathrm{p}<.05$ ]. A related-word advantage was shown by 16 of 22 third graders, sixth graders, and college subjects $(\mathrm{p}<.02$ by normal approximation to the binomial test).
Speed of processing. Letter search took longer than lexical decision for all age groups. As can be ascertained from Table 1, in no instance, within any grade level, was the mean RT for target lexical decision significantly longer than for target letter search. The pattern also held true for prime RTs, which were all neutral with respect to prior priming.

\section{Discussion}

Thus, in our study, semantic context effects were found both when semantic responses and nonsemantic responses were required to the prime. The levels of processing hypothesis predicted that semantic processing of the prime would yield contextual facilitation independently of the level of target processing and that nonsemantic prime processing would diminish or eliminate completely the semantic priming. But our findings need a different account. A modified levels of processing hypothesis, one which takes into account levels of processing of both stimuli, as well as relative speeds of processing semantic and nonsemantic codes, is needed to account for the facilitation found in the LS/LS and LS/LD conditions.

Semantic prime processing. When the prime was processed semantically, priming effects were found, as was predicted by the levels of processing hypothesis. Although M. C. Smith (1979) found facilitation effects when LS was the target task, M. C. Smith et al. (1983) argued that interference effects such as we found in our LD/LS condition support the levels hypothesis. The expectation for the LD/LS group, from M. C. Smith's levels of processing hypothesis, was that semantic prime processing would result in activation sufficient to facilitate target letter search. In M. C. Smith's (1979) study, semantic processing of the prime was passive; the stimulus was presented for a fixed duration with no response required. In our study, a lexical decision was required about the prime in the form of a response. Similar interference effects have been found with semantic judgments about the prime (Parkin, 1979) and naming responses to the prime (Henik et al., 1983, Experiment 3) followed by target color naming. Thus, when the target is processed nonsemantically, facilitation effects have been found when the prime is processed passively (target letter search condition in M. C. Smith, 1979; silent reading condition in M. C. Smith et al., 1983; Warren, 1974) and subjects therefore do not have to switch attention from a semantic level of processing of the prime to a nonsemantic level of processing of the target. There should be no cost of suppressing an expectancy for a related target, because no expectancy was generated consciously. On the other hand, in our LD/LS condition and the others listed above for which interference effects were found, subjects needed to switch their attention from a semantic to a nonsemantic level of analysis. In our study, this was indeed a difficult task (as demonstrated by slower letter search than in the LS/LS condition and by subjective reports, although not by error rates).

Nonsemantic prime processing. If we considered the results only of nonsemantic priming for college subjects 
in the LS/LS condition, we might have been content to accept M. C. Smith's (1979) levels of processing hypothesis. As in M. C. Smith's (1979) study, this condition in our study showed no context effect for these subjects. Smith contended that if the context effect is absent with dual letter search, as it was for the college subjects in her study and in ours, then two possibilities remain: (1) Letter search of the prime eliminates the priming effect (elimination hypothesis), or (2) letter search of the target interferes with the use of the semantic activation generated by the prime (use hypothesis). Her evidence that passive semantic processing of the prime facilitated target letter search left the use hypothesis in doubt, but our data in the nonsemantic prime conditions rendered the elimination hypothesis implausible.

If the lack of facilitation in the LS/LS condition had been due to interference with the use of semantic activation, then target $\mathrm{LD}$ should have picked up the advantage from semantic priming. M. C. Smith et al. (1983) and Henik et al. (1983) provided further evidence against the use hypothesis when they found no facilitation with prime letter search and target lexical decision (LS/LD). But in every grade in our LS/LD condition, a facilitation effect was found. Interestingly, it was the college data that were most consistent with the use hypothesis: A facilitation effect was found with a nonsemantic prime task when followed by a semantic target task, but not when followed by a nonsemantic target task.

Speed of processing. Furthermore, facilitation was found in the LS/LS condition in both third and sixth graders. These facilitation effects can be better understood by reference to the speed of processing hypothesis. Because letter search took longer than lexical decision, it may be argued that lexical access resulted even with the prime letter search, and therefore had the opportunity to facilitate target lexical decision for all grades and target letter search for the third and sixth graders. Because target letter search was relatively rapid for college subjects, it is possible that they did not have time to benefit from lexical access with their attention directed elsewhere.

The speed of processing component of the model is suggested even by the standard priming effect obtained when both prime and target are responded to with a lexical decision. Essentially, the strength of the effect decreased with increasing age. Morton (1969) and Meyer et al. (1975) predicted that context effects would be larger when stimulus words were more difficult to recognize. Stanovich et al. (1981) tested this hypothesis directly and confirmed it. Because the stimuli in our study were selected with familiarity to the younger children in mind, the words were no doubt easy for the college subjects to process. Hence, the context effects for these subjects should have been smaller. Compared to results of similar studies, the lexical decision times for college subjects were very short. Furthermore, the smaller context effects with increasing age are consistent with the interactivecompensatory theories of context effects (Stanovich, 1980). Smaller context effects have been found for older and better readers, who analyze words faster. West and Stanovich (1978) and Stanovich et al. (1981) have reported diminishing context effects from as high as $361 \mathrm{msec}$ for second graders to as low as $26 \mathrm{msec}$ for college students.

Summary. Thus, context effects were found with both semantic and nonsemantic prime processing, although the direction of the effects depended on the interaction of levels of processing of the prime and target stimuli. That the semantic information was available to affect target processing even with nonsemantic prime analysis may have been a function of the relative speeds of the two processes. Therefore, it is conceivable that in a nonsemantic task that can be performed at least as rapidly as a lexical decision task, semantic information may not be available early enough to result in context effects (speed hypothesis). We pursued this possibility in Experiments 2 and 3.

\section{EXPERIMENT 2}

Our speed of processing hypothesis is in part predicated upon speed differences that emerged from age comparisons in Experiment 1. We assumed that the letter and word codes were processed separately, thereby allowing them to interact with one another, and we assumed further that the interaction between these separate codes was possible at all three educational levels, with speed differences in part leading to the age effect. Posner (1978) has suggested that dimensional interactions of this kind can be explained by an "isolable subsystems" model: When there are multiple codes of information represented in a stimulus, then different pathways may be activated separately. If the information from one pathway is accessed earlier in time than the other, then this information is available to interact with (facilitate or interfere with) decisions to be made about the alternative code. Because we had already used a nonsemantic task that was more time-consuming than the semantic task, we now replaced the letter search task with a case judgment task. Subjects responded to stimuli presented in all upper- or all lowercase letters. Thus, we again required a decision that was not contingent upon lexical access but that took less time than a letter search (see Results). ${ }^{3}$

We know from Garner's (1978) study that there are many types of dimensional interaction possible. Because L. B. Smith and Kemler (1978) found in general that the processing of dimensions changes developmentally from integral to separable, it is conceivable that our age effects might be better understood in terms of changes in stimulus perception rather than in terms of levels or speeds of processing. Fortunately, as Garner demonstrated, it is possible to determine how subjects perceive the dimensions of a multidimensional stimulus from a set of discrimination, focusing, and classification tasks. Across these tasks, subjects are required to respond to different attributes of each stimulus in a set. With two-dimensional stimuli, simple discrimination tasks require classification on one of 
two dimensions (e.g., case or lexicality), with the other held constant. Discrimination with correlated dimensions requires classification on the basis of either of two dimensions, values of which are yoked. In focusing tasks, one class of stimuli is defined by the conjunction of two particular values; the other class comprises all remaining stimuli. In two classification tasks, the stimuli are partitioned by their values on one dimension, whereas the other dimension varies freely. The third classification task requires the biconditional classification of the four stimulus properties into two response categories. (Specific stimulus pairings in each numbered task are listed in Table 2.)

Thus, the simple discrimination tasks provide baseline data for the classification and focusing tasks. In the critical comparison, if either of the first two classification tasks takes longer than its respective simple discrimination task, this reveals that the dimension to be sorted was not attended selectively: Irrelevant dimension variation interfered with the sorting response. Thus, we have an alternative method of measuring context effects: We can measure the effect of semantic (lexical) information on nonsemantic (case) judgments, as well as the reverse interaction. When compared with RTs for the baseline discrimination tasks, RTs for the focusing tasks reveal whether the subject is able to focus on a particular stimulus, rather than on stimulus attributes within dimensions. If the discrimination task with correlated dimensions was processed faster than the simple discrimination task, this would indicate that the subjects were processing the two stimulus dimensions in an integral, rather than separable, fashion, because redundant information from the two dimensions would be better than information from either dimension alone. If this result was found for the younger subjects only, we would suspect that any differences in context effects might be due to the development of perceptual strategies. Finally, if the biconditional classification was the most rapid of the classification tasks, we would conclude that subjects were responding to a configural or emergent property of these stimuli, rather than to case or lexicality.

\section{Method}

Subjects. The subjects were 32 middle-class suburban third graders (mean age $=9.16$ years, $S D=0.53$ ), 32 sixth graders (mean age $=11.12$ years, $\mathrm{SD}=0.60$ ), and 32 college undergraduates, who received course credit for their participation.

Stimuli. The same sets of words and nonwords were used as described in Experiment 1. However, half of the stimuli were presented in uppercase and half in lowercase letters. There were equal percentages of upper- and lowercase stimuli for the different conditions.

Apparatus. Stimuli were presented on a Televideo 950 terminal controlled by a Northstar Horizon Z80A microcomputer. Stimulus presentation times and interstimulus intervals were controlled by the computer's $60-\mathrm{Hz}$ clock; RTs were measured by a Mountain Hardware 100,000-day clock (accuracy to $1 \mathrm{msec}$ ). Subjects responded via one of two predesignated keys on the detachable TVI 950 keyboard, allowing for accurate control of viewing distance.

Discrimination tasks. Each task (see Table 2) required discrimination between two of the four types of stimuli (uppercase words, uppercase nonwords, lowercase words, and lowercase nonwords). With six pairs of stimuli, there were six tasks: Four of these involved stimuli differing on a single dimension, case or lexicality, and two of these involved correlated or redundant dimensions.

Focusing tasks. For each of the four tasks, one stimulus type was to be discriminated from any of the remaining three stimulus types.

Classification tasks. There were three tasks involving classification of all four stimuli, two for each response: The classifications were based on case, lexicality, and the biconditional combination of the four stimulus classes.

Table 2

Mean Reaction Times (and Error Rates) by Grade Level and Task-Experiment 2

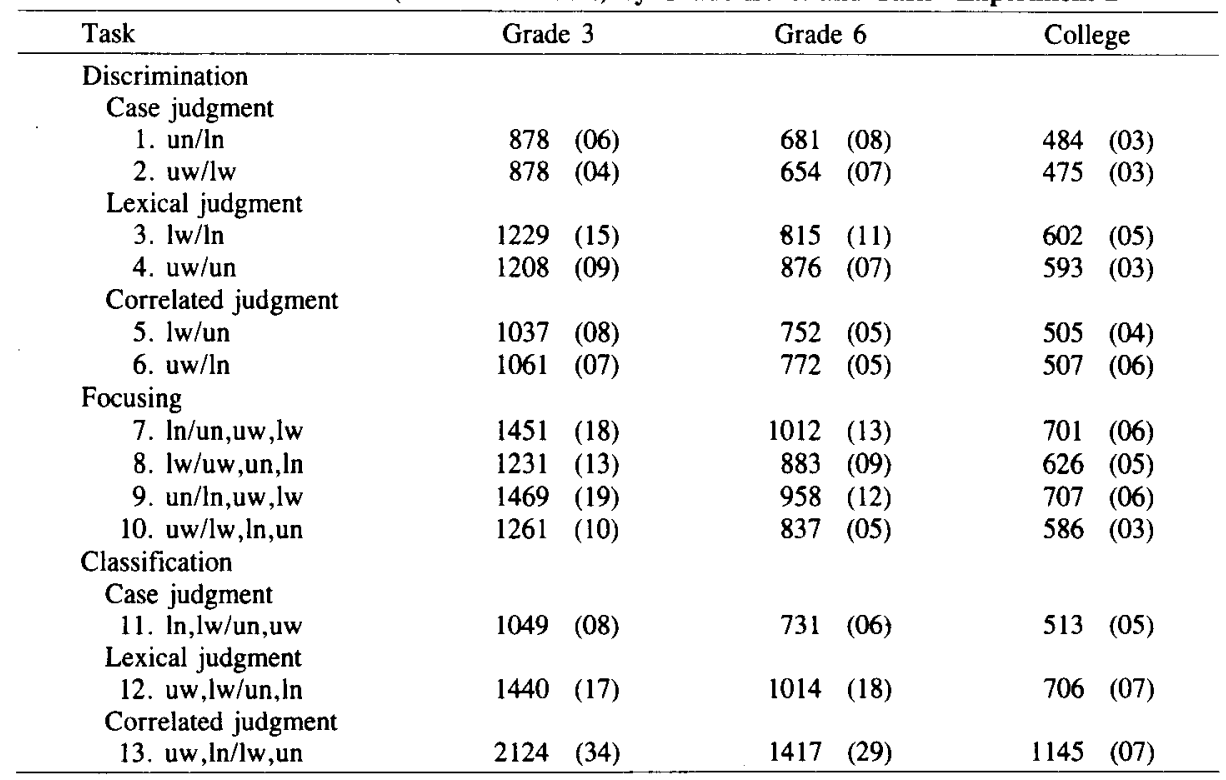

Note $-R T$ s in msec; error rates in parentheses to nearest hundredths, decimals omitted. $w=$ word; $n=$ nonword; $u=$ uppercase $; l=$ lowercase. 
Procedure. For each of the 8 subjects selected in each of the four conditions of the priming experiment $(\mathrm{N}=32$ for each grade; see Experiment 3), ${ }^{4}$ a different order of 13 tasks was determined pseudorandomly so that no one type of task was unequally represented in any position in the sequence. The 13 tasks were then presented in the predetermined sequence. For the college subjects only, the sequence then was administered in descending order, for a total of 26 tasks (13 given twice each, but with different sampling of stimuli for the descending order). In each task, 20 trials were administered. Prior to each task, instructions were presented on the screen and given orally by the experimenter. These included information about which key was to be used for which response(s). At any time during the task, a subject could press the space bar to retrieve the instructions.

Each task began with a central ready signal followed by presentation of a stimulus. The subject's response cleared the screen. A 1,000-msec ISI preceded the next stimulus.

\section{Results}

Perceptual analysis. Having raised the concern that context effects might be a function of changing perceptual analysis strategies across age, rather than of levels or speeds of processing the stimuli, we needed to identify the strategies of perceptual analysis at each grade level and compare these strategies across grades. Mean RTs and error rates are presented in Table $2 .^{5}$ The same patterns of RT were found at each grade level: Correlations between grades (across tasks) ranged from .98 to .99 . Patterns were similar for error rate, with the intergrade correlations ranging between .69 and .93 .

Selective attention. Selective attention to lexical information was evaluated by comparing the baseline discrimination RT (mean of Tasks 3 and 4) to its respective classification task RT (Task 12). Selective attention to case was evaluated similarly: the mean of Tasks 1 and 2 was compared to the mean of Task 11. These comparisons were significant at all grade levels. Lexical decisions were affected by case variability $[F(1,31)=20.11,43.15$, and 76.93 for subjects in Grade 3, Grade 6, and college, respectively, all ps $<.01]$. Case judgments were affected by lexical variability $[\mathrm{F}(1,31)=14.92,12.47$, and 13.98 for subjects in Grade 3, Grade 6, and college, respectively, all ps $<.01]$. For reaction time, case variability affected lexical decisions more than lexical variability affected case judgments, at least in sixth graders and college subjects [for sixth graders, $\mathrm{F}(1,31)=28.44$, $\mathrm{p}<.01$; for college subjects, $\mathrm{F}(1,31)=28.68, \mathrm{p}<.01$; for subjects in Grade 3, F $<1$ ].

Alternative perceptual strategies. If the stimulus dimensions were perceived as integral rather than separable, the correlated discriminations should have been faster than the simple discriminations. Although the mean RTs for Tasks 5 and 6 fell between the means of the case (Tasks 1 and 2) and lexical decision (Tasks 3 and 4) RTs, it can be argued that subjects were analyzing Tasks 5 and 6 as case judgments with interference from the lexical dimension: The case classification task (Task 11) took no longer than these correlated discrimination tasks.

Configural perception would have been indicated by the superiority of the biconditional classification; however,
RTs and error rates indicate that this clearly was the most difficult task at each grade level.

At each grade level, the focusing tasks requiring sorting by lower- or uppercase words (Tasks 8 and 10) took no longer than the lexical decision simple discrimination tasks (Tasks 3 and 4), indicating that subjects were able to focus on word stimuli of a particular case when making their lexical decisions (nonword focusing RTs were much longer). ${ }^{6}$

Relative speeds of processing. One aim of this experiment was to determine the relative speeds of processing of the two dimensions of case and lexical information. Therefore, we evaluated the baseline data from Tasks 1-4. At each grade level, case judgments (mean of Tasks 1 and 2) were made faster than lexical decisions (mean of Tasks 3 and 4) when there was no variation in the alternate dimension. These differences were significant $[\mathrm{t}(31)=7.99,8.16$, and 18.04 for subjects in Grade 3, Grade 6, and college, respectively; all ps $<.01$ ].

\section{Discussion}

Subjects in Grade 3, Grade 6, and college appeared to perceive the stimuli in the same manner, at least when sorting stimuli by the dimensions of case and lexicality. At no grade level (see Table 2) was there evidence of integrality or configurality. Rather, subjects were able to attend to both dimensions in question. They were not able, however, to selectively attend to either dimension completely. Although variation in either dimension affected decision times for the other dimension, the speed of processing hypothesis would appear to be valid: The easier-to-process case information interfered more with lexical decisions than did lexical information with case decisions. We would thus predict that fewer semantic context effects would be found in a priming experiment using these dimensions than were found in Experiment 1, because nonsemantic decisions could be made on the average before semantic information would be available to interfere.

\section{EXPERIMENT 3}

In the final experiment, the case judgments and lexical decisions studied in Experiment 2 were used in a contextual priming experiment, similar in most respects to Experiment 1 . Context effects were expected to be minimal when case judgment was the prime task, because case information would be available for a response before lexical access would result in priming.

\footnotetext{
Method

Subjects. The subjects were those who participated in Experiment 2.

Stimuli. The same sets of words and nonwords were used as described in Experiment 1. However, half of the stimuli were presented in uppercase and half in lowercase letters.

Apparatus. The same computer system was used as in Experiment 2.

Design. The same design was used as in Experiment 1, with the
} 
following exceptions: (1) case judgment (CJ) replaced letter search as the nonsemantic task; (2) for each of the five prime-target conditions, an equal number of trials comprised lowercase/lowercase, uppercase/uppercase, lowercase/uppercase, and upper case/lowercase combinations; and (3) "uppercase" (for case judgment) and "word" (for lexical decision) responses were assigned to the same hand-the right hand for half the subjects and the left hand for the other half at each grade level.

Procedure. The procedure was the same as in Experiment 1, except that no target letter appeared above the word/nonword stimuli and instructions for case judgment replaced those for letter search.

\section{Results}

Mean RTs and error rates ${ }^{7}$ for the correct responses to target stimuli for the related-and unrelated-word conditions for case judgment and lexical decision are presented in Table 3. These means include only trials for which the prime response was also correct.

Semantic prime processing. The $L D / L D$ condition is the only one for which results can be compared with those of Experiment 1. This comparison suggests some sample variation: The third and sixth graders in the present experiment responded faster, whereas the college subjects were somewhat slower. However, the context effect in Grade 3 subjects was a nearly identical $93 \mathrm{msec}[\mathrm{t}(6)=$ $5.04, \mathrm{p}<.01]$. The much smaller context effect of $21 \mathrm{msec}$ in sixth graders was not significant, but the 12msec effect in college subjects was identical to that in Experiment 1 .

Although a semantic prime task followed by a nonsemantic target task resulted in inhibition effects in Experiment 1 , this effect held only for subjects in Grade 6 in the present experiment $(\mathrm{t}=1.95, \mathrm{p}<.05$, one-tailed $)$.

Nonsemantic prime processing. As can be seen in Table 3 , context effects with a case judgment made of the prime were rather small (range: -37 to $+15 \mathrm{msec}$ ) and nonsignificant (by analysis of variance and the binomial test).

Speed of processing. Although case judgments were faster than lexical decisions on the average, not as strong

Table 3

Mean Reaction Times (and Error Rates) to Target Words by Grade Level and Condition-Experiment 3

\begin{tabular}{|c|c|c|c|c|c|c|c|}
\hline \multicolumn{2}{|c|}{ Task } & \multirow{2}{*}{$\begin{array}{c}\text { Grade } \\
\text { Level }\end{array}$} & \multicolumn{5}{|c|}{ Condition } \\
\hline Prime & Target & & \multicolumn{2}{|c|}{ Related } & \multicolumn{2}{|c|}{ Unrelated } & \multirow{2}{*}{$\frac{\text { Context* }}{+93 \ddagger}$} \\
\hline LD & LD & 3 & 897 & (04) & 990 & (04) & \\
\hline & & 6 & 629 & (03) & 650 & (03) & +21 \\
\hline & & C & 513 & (01) & 525 & (04) & +12 \\
\hline \multirow[t]{3}{*}{ LD } & $\mathrm{CJ}$ & 3 & 1231 & (11) & 1272 & (08) & +41 \\
\hline & & 6 & 1011 & (04) & 941 & (08) & $-70 \dagger$ \\
\hline & & C & 633 & (02) & 629 & (04) & -4 \\
\hline \multirow[t]{3}{*}{ CJ } & CJ & 3 & 963 & (08) & 926 & (06) & -37 \\
\hline & & 6 & 688 & (05) & 693 & (09) & +5 \\
\hline & & C & 464 & (02) & 468 & (04) & +4 \\
\hline \multirow[t]{3}{*}{$\mathrm{CJ}$} & LD & 3 & 1624 & (20) & 1600 & (23) & -24 \\
\hline & & 6 & 1175 & (03) & 1190 & (08) & +15 \\
\hline & & C & 761 & $(02)$ & 765 & (04) & +4 \\
\hline
\end{tabular}

Note-RTs in msec; error rates in parentheses to nearest hundredths, decimals omitted. $L D=$ lexical decision; $C J=$ case judgment; $C=$ college. ${ }^{*}$ Context effect $=$ unrelated $R T-$ related $R T . \dagger p<.05$. $\ddagger p<.01$. a case can be made as was predicted from Experiment 2 or as was found for letter search and lexical decision in Experiment 1. In particular, a comparison of the unrelated RTs of the LD/LD and CJ/CJ conditions shows that the case judgments were faster for Grade 3 and college subjects, but slower than lexical decisions for Grade 6 subjects.

\section{Discussion}

These small effects are consistent with the speed of processing hypothesis: When subjects are required to attend to a nonsemantic level of the prime, which may be processed before lexical access occurs, then no semantic priming advantage or disadvantage should be realized for target processing. If we take into account the grade-level differences in speed of processing, then perhaps the Grade 6 interference effect becomes more reasonable. If case judgments were slower than lexical decisions, then once again (as in LD/LS, Experiment 1) we would expect longer RTs for related words, since subjects would have difficulty switching attention from the primed lexical information that becomes available before the case judgment can be made.

\section{GENERAL DISCUSSION}

In this series of experiments, we examined the role that active processing of context plays in affecting the speed of processing of related or unrelated target words. We found that context effects were a function of (1) the level of processing of both the prime and target words; and (2) the relative speeds of processing of these prime and target words.

\section{Levels of Processing}

Our initial concern was with M. C. Smith's (1979) hypothesis that redirection of subjects' attention from a semantic to a nonsemantic analysis of the prime stimulus would be sufficient to eliminate the contextual facilitation effect. When she found this with college subjects, as did Henik et al. (1983) in their first experiment, at least one plausible explanation was that priming was contingent upon a semantic analysis of the prime. Without the semantic priming, no context effects were expected. Our attempt at replication (Experiment 1) revealed the limitations of this interpretation. Like Smith, we found no facilitation effect when college subjects performed a letter search of both prime and target stimuli. Yet subjects in Grades 3 and 6, who differed from the college subjects primarily in their word recognition speed, were able to benefit from semantically related word pairs even when conducting two consecutive letter searches.

As M. C. Smith (1979) noted, she failed to test the possibility that semantic processing had co-occurred with the letter search, but she stated that the task demands of dual letter search made the use of the semantic priming unnecessary. Our letter search-lexical decision (LS/LD) condition provided a direct test of this use hypothesis: 
When subjects at all grade levels performed the semantic decision following the nonsemantic decision, they still benefited from semantic relatedness. Given that attention was directed to a nonsemantic level, the most plausible hypothesis would seem to be that semantic priming took place and its output was compatible with the target task. Even though M. C. Smith et al. (1983) and Henik et al. (1983) both failed to find significant facilitation in a comparable condition, Henik et al. reported that 8 of the 14 subjects in their LS/LD condition showed a relatedness effect. In their Experiment 3, they reported small facilitation effects when letter search of the prime was followed by color naming of the target. Individual differences are indicated as well by a comparison of Parkin's (1979) failure to find a priming effect when subjects counted syllables in the prime with M. C. Smith et al.'s finding of a reliable 41-msec facilitation. We cannot say much at this time about the relative importance of task manipulations versus individual differences, but we hope that the issue of individual differences receives some attention in the future. Nevertheless, the attentional hypothesis, that nonsemantic prime processing eliminates the relatedness effect, needs to be tempered.

The Stroop-like interference effect found at all grade levels in Experiment 1 (and also in sixth graders, Experiment 3; Henik et al., 1983, Experiment 3) can be best understood by contrast with the facilitation effect found by M. C. Smith (1979) when her subjects conducted target letter search following passive prime processing. Her effect can be explained by the semantic access that is assumed to result from automatic word recognition by skilled readers. No attentional switch is required when subjects are asked to perform target letter search because there is no response required to the prime. But when a lexical decision is required prior to the letter search (or the prime is named, as in Henik et al., 1983), then an expectancy is generated. This switch of attention has a cost when the expected (or semantically related) stimulus appears but has no relevance to the required response. This target stimulus itself acts as an attention-getter, and attention must be redirected to the proper level required of a letter search-hence the cost. Underwood and Thwaites (1982) attempted to account for such differences in the direction of priming effects in terms of the stage of processing at which the context influences processing. If the effect precedes in time attention to a particular word, as in priming, then the encoding stage can be affected and we would expect facilitation. If the activation occurs simultaneously with attention, as with conscious strategies, then the decision stage is affected, and decision time is delayed.

\section{Speed of Processing}

As we have argued, speed of processing may play a major role in the determination of these and other context effects. When trying to understand context effects in Experiment 1, we might consider the hypothesis that semantic processing takes precedence and that semantic information is therefore available early in processing to interact with another dimension to be processed, for example, letter information. But it is equally plausible that semantic information was available earlier than letter information in this particular task, because the semantic task was in some sense easier than the letter search task. In Experiment 3 , however, the case judgment task appeared to be easier, and so case information was available prior to the semantic information. The same is true of the visual analysis condition used by M. C. Smith et al. (1983) in their Experiment 1. Hence, we would not expect semantic relatedness to be as great an influence as in the priming task of our Experiment 1, and results from our Experiment 3 and Smith et al.'s visual analysis condition confirmed our predictions.

To account for the reduced context effects, the attentional hypothesis provides an alternative view to the speed hypothesis. It is conceivable that case judgment or gross visual analysis (detecting a star before the word) of the prime draws attention away from the lexical analysis of the prime and therefore eliminates the semantic activation or the spread of activation that M. C. Smith (1979; M. C. Smith et al., 1983), and Henik et al. (1983) hold responsible for the priming effect. Because these tasks can be processed without attending to the word itself, even a slow nonsemantic prime task may not allow lexical access, even if there is ample time for the semantic analysis to follow the nonsemantic analysis during the relatively long ISI in the priming paradigm. Although we failed to find priming with fast case judgments of the prime, we found that case judgments were affected by lexicality when subjects at all grade levels in Experiment 2 had to sort stimuli by case. Here, semantic analysis did co-occur even with a fast nonsemantic task. Subjects were attending to the word. But in the priming paradigm, if semantic analysis did co-occur, it did not seem to be of sufficient strength to result in priming effects. Perhaps attention to the word lasted only as long as was required to conduct the case judgment, thereby cutting short the semantic analysis required to find priming of the target stimulus despite the additional time available.

In conclusion, further tests of the speed and attentional hypotheses may further elucidate the origin and nature of priming effects. In the meantime, it may be concluded once again that subjects' attention to a nonsemantic dimension of a word does not preclude the generation of priming effects in word recognition. Rather, semantic access may occur even with intentional nonsemantic processing of the prime, but the generation of context effects may depend on the relative timing of attention to and access of semantic and nonsemantic codes of the prime stimulus.

\section{REFERENCES}

Carroll, J. F., Davies, P., \& Richman, B. (1971). American Heritage Word Frequency Book. New York: Houghton Mifflin.

Craik, F. I. M., \& Tulving, E. (1975). Depth of processing and the retention of words in episodic memory. Journal of Experimental Psychology: General, 104, 268-294. 
Doenring, D. G. (1976). Acquisition of rapid reading responses. Monographs of the Society for Research in Child Development, 41(2), 1-54. Donnenwerth-Nolan, S., Tanenhaus, M. K., \& Seidenberg, M. S. (1981). Multiple code activation in word recognition: Evidence from rhyme monitoring. Journal of Experimental Psychology: $\mathrm{Hu}$ man Learning \& Memory, 7, 170-180.

Fowler, C. A., Wolford, G., Slade, R., \& Tassinary, L. (1981). Lexical access with and without awareness. Journal of Experimental Psychology: General, 110, 341-362.

Garner, W. R. (1978). Selective attention to attributes and to stimuli. Journal of Experimental Psychology: General, 107, 287-308.

HaYs, W. L. (1974). Statistics for the social sciences. New York: Holt, Rhinehart \& Winston.

Henik, A., Friedrich, F. J., \& Kellogg, W. A. (1983). The dependence of semantic relatedness effects upon prime processing. Memory \& Cognition, 11, 366-373.

MARCEL, A. J. (1983). Conscious and unconscious perception: Experiments on visual masking and word recognition. Cognitive Psychology, 15, 197-237.

Mayzner, M. S., \& Tresselt, M. E. (1963). Tables of single-letter and bigram frequency counts for various word-length and letter-position combinations. Psychonomic Monograph Supplements, 1(Whole No. 2)

Meyer, D. E., SchVaneveldt, R. W., \& Ruddy, M. G. (1975). Loci of contextual effects on visual word-recognition. In P. M. A. Rabbitt \& S. Dornic (Eds.), Attention and performance V. London: Academic Press.

Morton, J. (1969). The interaction of information in word recognition. Psychological Review, 76, 165-178.

PARKIN, A. J. (1979). Specifying levels of processing. Quarterly Journal of Experimental Psychology, 31, 179-195

Posner, M. I. (1978). Chronometric explorations of mind. Hillsdale, NJ: Erlbaum.

Post, T. A. (1979). Software control of reaction time studies. Behavior Research Methods \& Instrumentation, 11, 208-211.

Schvaneveldt, R., Ackerman, B. P., \& Semlear, T. (1977). The effect of semantic context on children's word recognition. Child $D e$ velopment, 48, 612-616.

Schwantes, F. M., Boesl, S. C., \& Ritz, E. G. (1980). Children's use of context in word recognition: A psycholinguistic guessing game. Child Development, 51, 730-736.

SMith, L. B., \& KemLer, D. G. (1978). Levels of experienced dimensionality in children and adults. Cognitive Psychology, 10, 502-532.

Sмгтн, M. C. (1979). Contextual facilitation in a letter search task depends on how the prime is processed. Journal of Experimental Psychology: Human Perception \& Performance, 5, 239-251.

Smith, M. C., Theodor, L., \& Franklin, P. E. (1983). The relationship between contextual facilitation and depth of processing. Journal of Experimental Psychology: Learning, Memory, \& Cognition, 9. 697-712.

STANovich, K. E. (1980). Toward an interactive compensatory model of individual differences in the development of reading fluency. Reading Research Quarterly, 16, 32-71.

Stanovich, K. E., \& WeSt, R. F. (1979). Mechanisms of sentence context effects in reading: Automatic activation and conscious attention. Memory \& Cognition, 7, 77-85.

Stanovich, K. E., West, R. F., \& Feeman, D. J. (1981). A longitudinal study of sentence context effects in second-grade children: Tests of an interactive-compensatory model. Journal of Experimental Child Psychology, 32, 185-199.

UNDERWOOD, G., \& ThWAites, S. (1982). Automatic phonological coding of unattended printed words. Memory \& Cognition, 10, 434-442.

WARREN, R. E. (1974). Association, directionality, and stimulus encoding. Journal of Experimental Psychology, 102, 151-158.

WEST, R. F., \& STANOVICH, K. E. (1978). Automatic contextual facilitation in readers of three ages. Child Development, 49, 717-727.

\section{NOTES}

1. Although different words were used for the related and unrelated word pairs, a pilot study indicated that there were no latency differences between words in the different word pair conditions on a simple lexical decision task, suggesting that the words were of comparable difficulty. Since the same word and nonword pairings were used in each between-subjects condition, significant effects of prime and target task cannot be attributed to item variability.

2. Error rates in general were low for subjects in all grades (see Table 1). For Grade 3 subjects, error rates were a function of all three experimental factors interactively [prime $\times$ target $\times$ relatedness effect; $F(1,27)=6.95, p<.04]$. The error rate was higher for unrelated word targets $(.085)$ than for related word targets $(.065)[\mathrm{F}(1,27)=6.0$, $p<.03$ ]; although this effect is small, it suggests that the priming effect is shown in error rates as well as latencies. The three-way interaction was due to equivalent error rates in the LD/LD and LS/LS groups and higher unrelated-target error rates in the mixed-task groups in general, with the highest error rate found in the LS/LD group. A similar pattern held for Grade 6 subjects, with higher error rates for unrelated targets $[F(1,28)=11.83, p<.002$ for the relatedness main effect] and higher error rates for mixed-task groups than for same-task groups $[\mathrm{F}(1,28)=6.8, \mathrm{p}<.02$ for the prime $\times$ target interaction] For college subjects, error rates were uniform across cells, with no significant effects. Thus, at no grade level was there evidence of higher error rates for lower mean RTs.

3. A further advantage of the case judgment (CJ) task is that the same stimuli were used for both the case and lexical decisions. In the letter search task, the target letters were presented in addition to the prime or target stimuli, so there was the possibility of lateral masking effects (Smith, 1979) or altered attentional strategies.

4. The priming experiment (Experiment 3 ) actually was run before Experiment 2, so that sorting instructions would not influence performance on the priming task. We have presented the sorting study first for logic of exposition.

5. Since this set of tasks was given after the priming experiment, it would have been possible for response type to influence the results. However, this did not occur: there were no differences among groups on these tasks. Therefore, the data for all 32 subjects in each grade are presented.

6. The focusing results suggest a caution in comparing the speed of case and lexical judgments: Since the simple lexical discriminations are collapsed across word/nonword judgments, the true latency difference between case judgments and lexical decisions is probably less than the differences indicated by comparing Tasks 1 and 2 with Tasks 3 and 4 . For example, the focusing tasks suggest that word decisions may be made on the order of $200 \mathrm{msec}$ more quickly than nonword decisions, as compared with a simple discrimination difference of $340 \mathrm{msec}$ for Grade 3 subjects. Nevertheless, case judgments appear to be made more quickly than lexical decisions.

7. The only significant effect found for Grade 3 subjects was for the prime $\times$ target effect $[F(1,28)=10.92, p<.01]$. The CJ/LD tasks produced very high error rates. In Grade 6, only the relatedness main effect was significant $[F(1,28)=7.62, p<.01]$, with related words (.04) responded to more accurately than unrelated words (.07). Similarly, for college subjects, only the relatedness effect reached significance, with related words $(.02)$ responded to more accurately than unrelated (.04) words.

(Manuscript received September 7, 1984; revision accepted for publication July 5,1985 .) 